\title{
Effects of anesthesia \& anesthetic techniques on cellular immunity
}

Volume 7 Issue 6 - 2017

\section{Introduction}

An increasing body of evidence shows that the choice of anesthetic can strongly influence more than just the quality of anesthesia. The body's stress response to surgery first became a subject of interest in the 1920s, when David Cuthbertson observed that surgical patients had a large increase in urinary muscle breakdown metabolites postoperatively. Since then, studies have looked into detailing the stress response and whether it can be altered to the patient's advantage. Anesthetic choices can also have much wider implications, affecting cancer cell biology and its progression toward metastasis and invasion. This review will be looking specifically at the effects of regional anesthesia (RA) compared to general anesthesia (GA) with intravenous and volatile reagents, and the impacts of both of them on altering the surgical stress response, postoperative organ function and cancer progression. ${ }^{1}$ A clear definition of innate and adaptive immunity must be started with.

\section{Innate and adaptive immunity}

The immune system plays a vital role in survival by protecting us from the many potentially deadly infectious pathogens in our environment, as well as from cancer cells. The immune system is able to recognize pathogens and trigger their elimination through innate and then adaptive immune responses. ${ }^{2}$ Innate immunity, also called natural or native immunity, is the first line of defense and refers to protective mechanisms that are present even before infection. Its principal components are the epithelial membranes (which block pathogen entry), phagocytic cells (neutrophils and macrophages), dendritic cells, natural killer (NK) cells and several plasma proteins, including the complement system. The most important cellular reaction of innate immunity is inflammation - the process, mediated by dendritic and NK cells, whereby phagocytic cells are recruited and activated to eliminate aggressor agents. ${ }^{2}$

Adaptive immunity, also called specific or acquired immunity, consists of mechanisms that are induced by the recognition of specific pathogen antigens. The adaptive immune system is mediated primarily by lymphocytes, and its function can be classified into two types: humoral immunity, mediated by B-lymphocytes and their secreted antibodies; and cell-mediated or cellular immunity, mediated mostly by T-lymphocytes and their cytokines, which play an important role in immune cell activation, regulation, and communication. ${ }^{2}$

Besides its role in host defense against infectious agents and tumor cells, the inflammatory response is essential for tissue reconstitution after injury caused by accidental or surgical insults. Dysregulation of this inflammatory process may increase susceptibility to infections, accelerate the growth and metastasis of residual cancer cells, and result in postoperative complications, such as wound healing disturbances and infections leading to sepsis followed by multiple organ failure and death. ${ }^{2}$

\author{
Etedal Aamri,Amani Basnawi \\ Consultant Anesthesiologist, Saudi Arabia
}

Correspondence: Etedal Aamri, Consultant Anesthesiologist, High Risk OB Anesthesia KFSHRC, Riyadh, Saudi Arabia, Email eaamri@kfshrc.edu.sa

Received: April 22, 2017 | Published: April 26, 2017

\section{Effects of general anesthesia on inflammation}

The immune-modulating effects of anesthetics in vitro were first demonstrated more than 100years ago. The increasing knowledge of recent years is strongly related to developments in basic science and improvements in laboratory technique, e. g., cell separation and cell culture methods. It has been demonstrated that, at concentrations used clinically, different anesthetics depress the functions of the inflammatory response differentially. ${ }^{2}$ Here is a review of the effects of common anesthetic drugs on the immune system ${ }^{7}$ (Table 1).

Table I

\begin{tabular}{lll}
\hline Classification of Anaesthetics & \\
\hline General Anaesthetics & \multicolumn{2}{c}{ Local Anaesthetics } \\
\hline Intravenous Anaesthetics & Inhalational & Anaesthetics \\
\hline Ketamine & Halothane & Lidocaine \\
Thiopental & Sevoflurane & Bupivacaine \\
Propofol & Desflurane & Levobupivacaine \\
Fentanyl & Isoflurane & \\
Remifentanil & Enflurane & \\
Midazolam & & \\
Opioids & & \\
\hline
\end{tabular}

Intravenous anesthetic agents: Melamed et al., ${ }^{3}$ investigated the effect of intravenous anesthetic agents on cellular immunity. They injected rats with tumor cells and subsequently subjected the animals to different anesthetic agents. Rats treated with ketamine had 5.5 times the number of tumor cells of control rats. Rats treated with thiopental had 2 times the number of tumor cells of control rats. Melamed et al., ${ }^{3}$ also found that ketamine, thiopental, and propofol treatments suppressed NK cell activity and NK cell levels compared with the controls. They found a correlation between the number of viable tumor cells present at autopsy and NK cells in the aggregate of the groups but not in the individual groups. ${ }^{3}$ 
The link between docosahexaenoic acid (DHA) and eicosapentaenoic acid (EPA) and the low incidences of several strains of cancer has also been investigated. Siddiqui et al tested propofoldocosahexaenoate (propofol-DHA) and propofol-eicosapentaenoate (propofol-EPA) for their effects on the migration, adhesion, and apoptosis of MDA-MB-231 breast cancer cells. The propofol conjugates inhibited cellular adhesion, migration, and apoptosis in MDA-MB-231 breast cancer cells. ${ }^{3}$

A study was done on the effects of morphine and tramadol on pain and immune function during the postoperative period in 30 patients undergoing abdominal surgery for uterine carcinoma. Phytohemoagglutinin-induced T lymphocyte proliferation and natural killer cell activity were evaluated immediately before and after surgery, and $2 \mathrm{~h}$ after the acute administration of either $10 \mathrm{mg}$ of morphine IM or $100 \mathrm{mg}$ tramadol IM for pain. In all patients, phytohemagglutinininduced lymphoproliferation was significantly depressed by surgical stress. However, in the morphine-treated group, proliferative values remained lower than basal levels for $2 \mathrm{~h}$ after treatment, whereas in tramadol-administered patients proliferative values returned to basal levels. Natural killer cell activity was not significantly affected by surgery nor by morphine administration, whereas tramadol significantly enhanced the activity of natural killer cells. Both drugs produced a comparable reduction in postoperative pain. The study concluded that, as previously observed in the experimental animal, tramadol and morphine, when administered in analgesic doses, induce different immune effects. ${ }^{4}$

Using flow cytometry, another study was done to show whether propofol anaesthesia or isoflurane anaesthesia had more effect on the decrease in the Th1/Th2 ratio after surgery in patients undergoing craniotomy. The Th1/Th2 ratio decreased significantly after isoflurane anaesthesia, while it did not change after propofol anaesthesia. The ratio was significantly lower with isoflurane than propofol. Propofol anaesthesia attenuated the surgical stress-induced adverse immune response better than isoflurane anaesthesia. ${ }^{5}$

A comparison study was done on the effects of various anesthetics on natural killer (NK) cell activity and on resistance to experimental metastasis. It also studied mediating mechanisms and prophylactic measures. Fischer 344 rats served as controls or were anesthetized for $1 \mathrm{~h}$ with ketamine, thiopental, halothane, or propofol. Anesthetized rats were either maintained in normothermia or left to spontaneously reach 33 degrees and 35degrees. Rats were then injected IV with MADB106 tumor cells, and $24 \mathrm{~h}$ later lung tumor retention was assessed, or 3 weeks later, lung metastases were counted. Additionally, the number and activity of circulating NK cells were assessed after anesthesia. All anesthetics, except propofol, significantly reduced NK activity and increased MADB106 lung tumor retention or lung metastases. Hypothermia had no significant effects. Ketamine increased metastasis most potently, and this effect was markedly reduced in rats pretreated with a beta-adrenergic antagonist (nadolol) or with chronic small doses of an immunostimulator (polyriboinosinic:polyribocytidylic acid). Overall, the marked variation in the NK-suppressive effects of anesthetics seems to underlie their differential promotion of MADB106 metastasis. Prophylactic measures may include perioperative immunostimulation and the use of beta-blockers. ${ }^{9}$

Inhalational agents: Halogenated volatile anesthetics have been shown to have properties of immunomodulation and to suppress the function of NK cells. Specifically, isoflurane and halothane each attenuate the interferon stimulation of NK cells in mice. In addition, 1 in vitro investigation has yielded results that warrant further investigation of the effects of inhalational agents on immunosuppression. This study has demonstrated that sevoflurane administration leads to the altered release of cytokines such as IL-1 $\beta$ and tumor necrosis factor (TNF)- $\alpha$ by NK and NK-like cells. ${ }^{3}$ The identification and characterization of the immunosuppressive effects of inhalational agents has been challenging for researchers because of the many variables in the conditions of inhalational anesthesia administration and the different drugs to which patients are exposed. Figure 1 shows some of the direct immune modulations by volatile anesthetics. ${ }^{11}$ In a large retrospective analysis, Schlagenhauff et al found a relationship between anesthesia and cancer survival rate: compared with the use of local anesthesia, general anesthesia was associated with a decreased survival rate for patients with primary melanoma excisions. ${ }^{3}$

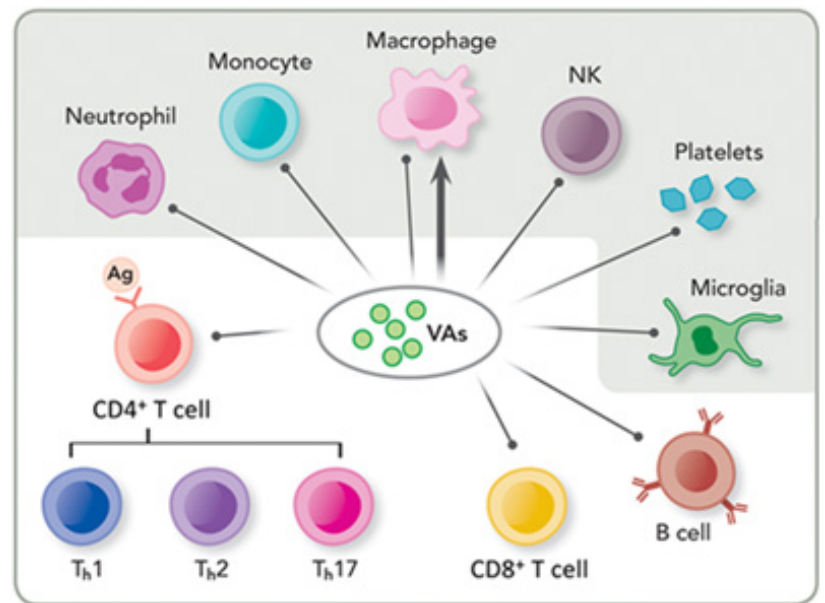

Figure I Direct immune modulations by volatile anesthetics (VAs). Depicted here are immune cells responsible for the innate (shaded) and adaptive (unshaded) immunity.VAs have been shown to suppress innate immunity by impairing or suppressing neutrophil adhesion, monocytes, macrophages and natural killer (NK) cells and affecting resident cells in tissues, such as platelets and microglial cells. VAs also suppress adaptive immunity by decreasing lymphocyte proliferation, such as cluster of differentiation (CD) 4 positive (CD4+) and 8 positive (CD8+) T cells as well as B cells. Note that VAs can have both inhibition (shown as a line with a dot) and potentiation (shown as a line with an arrowhead) effects on macrophages, depending on the site of infection or inflammation.Ag:Antigen;Th:T helper cell type I;Th2:T helper cell type 2; Th I7:T helper cell type 17.

Another study indicated that general anesthesia leads to the reduced circulation of NK cells in patients undergoing elective orthopedic surgery. Also, previous literature has suggested an association between cancer surgery and the systemic release of tumor cells and micrometastases. Further research has yielded data from both in vitro evaluations and in vivo animal studies that indicate impairment of neutrophils, macrophages, dendritic cells, and $\mathrm{T}$ cells with the use of anesthesia for cancer surgery. ${ }^{3}$ Moreover, another study showed that, in the clinical setting of a low stress procedure, the use of isoflurane anesthesia apparently increases cortisol level, this phenomenon was associated with less favorable metabolic and immune response when compared with sevoflurane or halothane. Further studies are required to verify whether sevoflurane anesthesia might maintain such advantages in more critical surgical procedures on severely ill, immunocompromised and/or malnourished patients. ${ }^{8}$

Nitrous oxide: The toxicity of nitrous oxide has been demonstrated. Nitrous oxide disrupts the process of DNA synthesis and depresses neutrophil chemotaxis. Additionally, nitrous oxide administration is associated with both depressed neutrophil function and reduced mononuclear cell production. ${ }^{3}$ 
The mouse model has revealed the possibility of immunosuppression from nitrous oxide administration; Shapiro et al demonstrated that nitrous oxide exposure is associated with the accelerated development of lung and liver metastasis. ${ }^{3}$

Nitrous oxide has also been examined for its effects on humans. Nitrous oxide exposure may increase bowel distension in patients who are subject to elective colon resection. However, further investigation has led to the interpretation that nitrous oxide does not increase the incidence of surgical wound infection. In addition, a follow-up of a randomized controlled trial indicated that nitrous oxide may not increase the risk of cancer recurrence after colorectal surgery. ${ }^{3}$

\section{Effects of regional and local anesthetics on inflammation}

Local anesthetics: The effects of local anesthetics on tumor suppression have been studied in cells. Lidocaine seems to exert an antitumor effect. Ropivacaine seems to exert a suppressive effect on the growth of tumor cells. ${ }^{3}$ Lidocaine possibly exerts its antiproliferative effect on tumors through the epidermal growth factor receptor (EGFR). An experiment was structured such that lidocaine was administered in clinical concentrations to tongue cancer cells with the concurrent evaluation of EGFR levels of activity. The clinical concentration of administered lidocaine led to the marked decrease of EGFR-induced proliferation of tongue cancer cells and to the inhibition of the EGFRstimulated tyrosine kinase activity that stimulates EGFR. ${ }^{3}$

The effect of ropivacaine on cancer cell growth has been studied in vitro. Ropivacaine seems to suppress the growth of human colon adenocarcinoma cells and has been demonstrated to inhibit cancer cells in a dose-dependent manner. Although more research is needed to elucidate the effects of local anesthetics on cancer cell growth, these studies show promise for optimized approaches. ${ }^{3}$

Regional anesthesia: More studies on the effect of regional anesthesia on immunosuppression are needed. To date, most of the studies on the effect of regional anesthesia on immunosuppression are retrospective. One study examined the incidence of biochemical cancer recurrence in 2 treatment groups after open prostatectomy under general anesthesia. One group received open prostatectomies with epidural analgesia and the other group was given postoperative opioid analgesia. The group with epidural analgesia had a $57 \%$ reduction in cancer recurrence compared to the opioid group. ${ }^{3}$

An investigation by Wada et al. explored the possibility that general anesthesia may improve the overall treatment of cancer. In a rat model, sevoflurane general anesthesia and laparotomy each suppressed tumoricidal function in liver mononuclear cells, and spinal block attenuated this suppressive effect. The Wada et al study also explored the possibility that the combined administration of sevoflurane and a spinal block may reduce the promotion of tumor metastasis. $^{3}$

Regional anesthesia may exert an effect on breast cancer metastasis. In one investigation, serum samples were taken from breast cancer surgery patients who experienced various anesthetic techniques. These samples were studied for breast cancer cell function in vitro, and the results indicated a possible link between anesthetic technique and breast cancer cell function. ${ }^{3}$ Regional anesthesia influences the long-term outcome of cancer surgery in 3 ways. First, regional anesthesia may attenuate the intrinsic immunosuppression from surgery. Second, patients who receive regional analgesia often do not need as much opioid treatment, and as a result, tend to avoid the immunosuppressive effects that accompany opioid treatment.
Third, the combination therapy of regional and general anesthesia leads to a reduction in the dose of inhalational anesthetic required. This decrease in the required dose can potentially affect long-term outcome from cancer-related surgery. ${ }^{3}$

\section{Basic science findings related to neuraxial and regional anesthesia}

Reduced blood loss, decreased need for blood transfusion, superior analgesia, and increased mobility have all been cited as advantages of regional anesthesia over systemic opioids. ${ }^{3}$ In addition, neuraxial and regional anesthesia and analgesia provide a substantial reduction in postoperative pain, intravenous opioid use, and volatile anesthetic requirements. Both the attenuation of the surgical response and the inhibition of the immune response as a result of the use of either epidural analgesia or anesthesia have been documented in a study by Hong and Lim. Only a few studies have been published that demonstrate the effect of neuraxial and regional anesthesia on the immune system. Even fewer studies explore the effect of neuraxial and regional anesthesia on the immune system and cancer recurrence. ${ }^{3}$

Studies have illustrated the effects of regional anesthesia on cytokine serum levels Il-2, IFN- $\gamma$, Il-10, and plasma epinephrine/ cortisol. Plasma levels of cortisol and epinephrine were shown to be significantly decreased in the regional anesthesia group. IFN- $\gamma$ also was shown to increase as a result of the administration of regional anesthesia. A measurement of T-helper cells and lymphocytes revealed that the regional anesthesia group had significantly higher numbers of T-helper cells and lymphocytes postoperation compared to the group without regional anesthesia. Another in vitro study of 32 people demonstrated the effect of regional anesthesia on TNF- $\beta$ and vascular endothelial growth factor (VEGF). TNF- $\beta$ and VEGF are important markers for inflammation. Although the sample group in this study was small, in terms of the effect of regional anesthesia, it illustrated both a postoperative fall in VEGF and also a postoperative increase in TNF- $\beta .^{3}$

Both of these in vitro studies share a theme that merits further study: regional anesthesia attenuates the immunosuppressive response to surgery. Different studies conflict with regard to the effects of morphine on tumor progression. One study demonstrated that morphine treatment leads to a reduction in colon adenocarcinoma cells. Different receptors for endogenous and exogenous opioids possibly cause divergent effects for in vivo animal studies and in vitro assays. $^{3}$

Normal volunteers have also shown that morphine administration leads to the suppression of antibody-dependent cell cytotoxicity. However, the effect of morphine on NK and B cells remains unknown. Possible mechanisms by which morphine exerts its effects are explored in Figures $2 \& 3$, reprinted from Gach et al.. The opioid-cancer recurrence associations found in animal studies and in human in vitro studies have increased interest in the use of regional anesthesia and analgesia in patients with cancer. In the past decade, several retrospective trials have shown mixed results regarding the relationship between opioids and the stimulation of both metastasis and immunosuppression. ${ }^{3}$

\section{Clinical implications of the anti-inflammatory properties of anesthetics}

Investigations of the immune effects of anesthetics have been derived mostly from in vitro studies, because human clinical studies are more complex, involving such variables as type and duration of surgery and patient complications. Although it is difficult to 
distinguish the relative contributions of surgical stress, anesthetics and analgesic agents to the patient's immune system, anesthesiologists must not ignore the immunosuppressive effects of anesthetic drugs on perioperative immunity. ${ }^{2}$

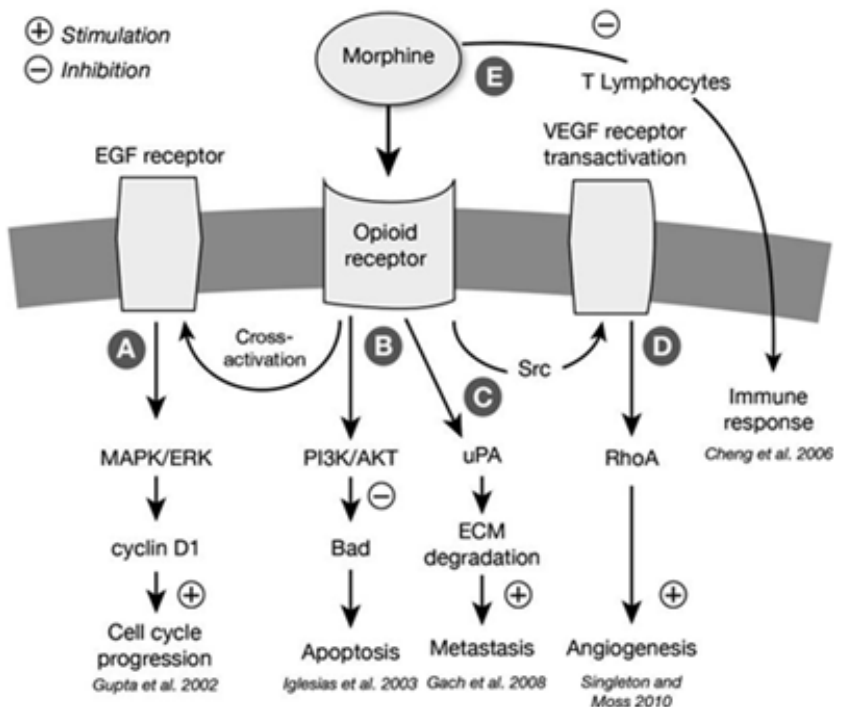

Figure 2 Possible mechanisms of the opioid receptor-mediated influence of morphine on tumor growth. Morphine binds to the $\mu$-opioid receptor and (I) stimulates the mitogen-activated protein kinase (MAPK) signaling pathway via the $G$ protein-coupled receptors/ extracellular-signal-regulated kinase (ERK) pathway, resulting in cell cycle progression; (2) activates the phosphatidylinositol 3-kinase/AKT (PI3K/AKT) pathway, mediating antiapoptotic effects through the $\mathrm{Bcl}-\mathrm{xL} / \mathrm{Bcl}$-2-associated death promoter protein; (3) upregulates urokinase plasminogen activator (UPA) expression and secretion promoting extracellular matrix (ECM) degradation and metastasis; (4) transactivates vascular endothelial growth factor (VEGF) receptors and induces angiogenesis; and (5) suppresses the function of T lymphocytes, leading to immunosuppression. (Figure and caption reprinted with permission from Gach et al.,"' EGF: Epidermal Growth Factor; Src: Sarcoma.

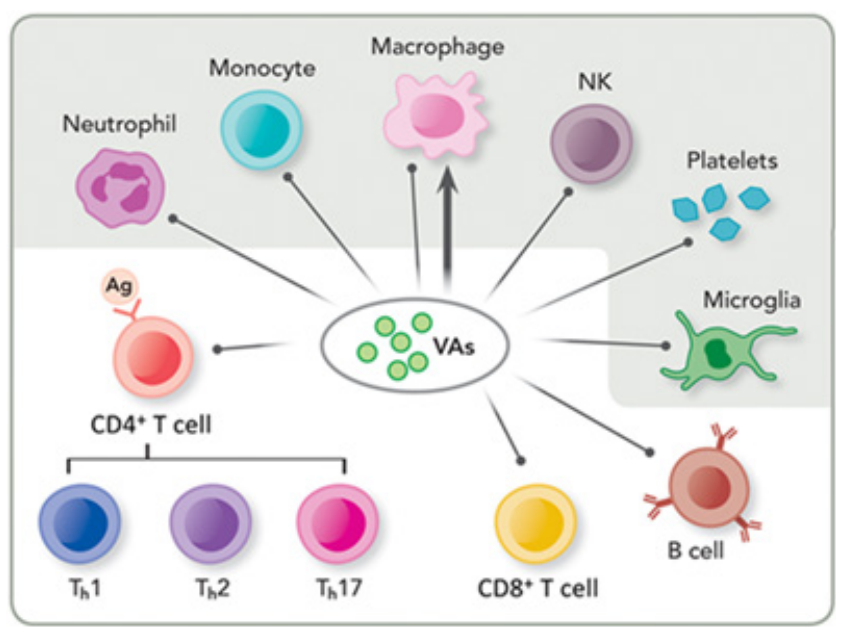

Figure 3 Possible pathways, other than through the opioid receptors, by which morphine influences cancer progression and suppression. (Figure and caption reprinted with permission from Gach et al.,"

Bax: bcl-2-associated x protein; Bcl-2: B Cell Lymphoma 2; Bim: bcl2-interacting mediator of cell death; COX-2: Cyclooxygenase-2; ECM: Extracellular Matrix; Fas:TNF receptor superfamily member 6; IL: Interleukin; JNK: c-Jun N-terminal Kinase; MAPK: Mitogen-Activated Protein Kinase; MMPs: Matrix Metalloproteinases; NK: Natural Killer (cells); NO:Nitric Oxide; PGE-2: Prostaglandin E2; ROS: Reactive Oxygen Species;TNF:Tumor Necrosis Factor;VEGF:Vascular Endothelial Growth Factor.

\section{In the Perioperative period}

Surgery is a major traumatic element in postoperative immunodepression in normal people. A higher degree of surgical trauma determines greater immunodepression. ${ }^{10}$ Figure 4 shows a schematic diagram of possible modulators of immune competence during anesthesia and surgery. ${ }^{2}$ Surgical trauma induces an endocrine, metabolic, hemodynamic and immune response that persists for at least a few days. It is generally accepted that the effect of anesthetics on the immune system is modest compared to the effects induced by major surgery or trauma; thus, anesthetics may not have any clinically significant effects on immune function in healthy patients anesthetized for short procedures, in whom the inflammatory response is usually balanced, well controlled and of limited duration. An immunosuppressive effect of approximately $20 \%$ might not have great consequences for an immunocompetent patient. However, if the patient has a genetic predisposition to immune impairment or is already compromised, e. g., by aging, tumor burden, diabetes mellitus or malnutrition, the immunosuppressive effects of anesthetics might play a salient role in postoperative infectious complications, morbidity and mortality. In addition, an increasing number of immunosuppressed elderly patients require anesthesia and intensive care treatment. Thus, the possible immunomodulatory effects of anesthetics must be widely comprehended and agents chosen wisely. Particularly in patients with cancer, immunosuppression after surgery accelerates the growth of residual malignant cells and promotes the establishment of new metastases. On the other hand, the immunosuppressive effects of anesthetics that lead to anti-inflammatory responses may be therapeutically beneficial in distinct situations such as ischemiareperfusion injury, the systemic inflammatory response syndrome, or acute respiratory distress syndrome (ARDS). Therefore, the effects of anesthetics on immunity may be not only adverse but also beneficial on the prognosis of specific patients. The immune effects of surgery and anesthetics affect the long-term outcomes of patients after surgery. Therefore, awareness of these immunological properties is helpful for daily anesthetic management. ${ }^{2}$

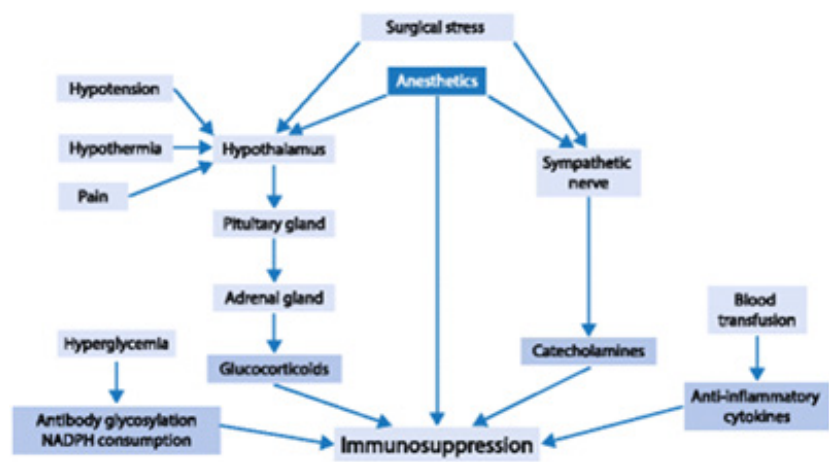

Figure 4 Schematic diagram of possible modulators of immune competence during anesthesia and surgery. Anesthetics have direct effects on the immune system and indirect effects through neuro-immune-endocrine interactions during surgical stress.

In brief, the effect of anesthetics on the immune system has been less investigated in vivo than in vitro; current findings are contradictory and indicative of only minor clinical importance. At present, no one mode of anesthesia can be recommended in favor of another in terms of effect on the inflammatory response. ${ }^{2}$

In addition to effects on non-specific cell-mediated immunity, some local anesthesia techniques can inhibit parts of the neuroendocrine response to surgery. Subarachnoid and epidural blockade blunts the increase in plasma levels of epinephrine, norepinephrine and cortisol 
induced by surgery. The reduction in the neuroendocrine stress response is most pronounced in connection with surgical procedures on the lower abdomen and lower extremities. In these cases, epidural anesthesia can provide complete blockade of all afferent neurogenic stimuli from the surgical field, reducing the stress-related response, and can even reduce perioperative mortality and postoperative morbidity after some surgical procedures. ${ }^{2}$

\section{In the Intensive care unit}

While a slight immunosuppressive effect of anesthesia during surgery is probably of minor importance because of the limited duration of exposure, critical care settings pose a different set of challenges. In the ICU, patients are often exposed to anesthetic agents for several days, and the side effects of immunosuppression have been shown to be clinically important in this population. In this context, as early as 1956, bone-marrow depression was described after prolonged ventilation with nitrous oxide.

Later, in 1983, increases in ICU mortality were reported after the introduction of etomidate for sedation. Patients sedated with etomidate had a mortality of $77 \%$, versus only $28 \%$ in patients not given this agent. It was later discovered that etomidate inhibits the synthesis of cortisol precursors and thus reduces levels of plasma cortisol. A high rate of infections has also been described in ICU patients receiving long-term thiopental infusions. ${ }^{2}$

Propofol or benzodiazepines are most often used for long-term sedation in the ICU. Animal studies have shown reduced defense against infection following long-term infusion of propofol as well as benzodiazepines. A single study has shown increased plasma levels of proinflammatory cytokines (IL-1, IL-6, TNF- $\alpha$ ) following long-term propofol infusion, while a reduction in proinflammatory cytokines was observed following benzodiazepine infusion. To avoid a potential detrimental effect of long-term sedation in critically ill patients, the duration and the doses of sedative agents should be kept as low as possible, not least because these patients are immunocompromised. Daily wake-up trials are recommended to reduce the risk of oversedation. $^{2}$

\section{Influence of anesthetic type on cancer outcomes and recurrence}

Previous research indicates that the choice of anesthetic technique may have an effect on cancer progression by influencing cell invasion, migration, proliferation and metastasis. Clinical studies indicate that RA and/or intravenous GA can be preferable for cancer patients in comparison to volatile GA, with regard to outcomes. Breast cancer patients who underwent surgery with a paravertebral nerve block in combination with GA have a lower incidence of cancer recurrence or metastasis than those undergoing surgery with GA and patientcontrolled morphine analgesia. Another study has shown that the recurrence rate of prostate cancer after prostatectomy under GA with epidural analgesia was significantly lower than that under GA with postoperative opioid analgesia. In addition, patients with ovarian adenocarcinoma who underwent surgery with epidural anesthesia and analgesia had better long-term outcomes than those who were given only GA, and excision of melanoma under GA has been associated with a decreased survival rate when compared to patients who had excision under a local anesthetic. Furthermore, a retrospective study, reporting the data from 2838 patients registered for surgery for breast, colon, or rectal cancer, showed that propofol anesthesia might be better than sevoflurane in surgery for some cancer types for 1-year survival, however, after adjustment for several confounders, the observed differences were not statistically significant. ${ }^{1}$

\section{Effect of anesthetic choice on anticancer immunity}

Cell-mediated immunity with T-helper 1 (Th1) cells and NK cells is considered an important mechanism in enabling the body to act against cancer. It has been associated with reduced risk of cancer recurrence after surgical resection, because NK cells are reported to have cytolytic activity against cancer cells. Volatile GA has been shown to suppress this anticancer immunity. It has also been reported that NK cells target cancer cells and prevent metastases, which is supported by evidence showing a correlation between higher recurrence rates and reduced NK cell activity. NK cell cytotoxicity is decreased by all types of anesthesia, but GA in particular appears to have a greater effect. Ketamine, thiopental, and halothane reduced NK cell activity and increased tumor retention and metastasis. Propofol has been shown to have protective effects through a variety of mechanisms that enhance antitumor immunity. A possible reason for this is that intravenous GA, when compared to volatile GA has been shown to increase IL10 , which is known to have antitumor activity and help with healing and repair. Epidural anesthesia in combination with GA, compared to GA alone, has been shown to increase the ratio of Th1 cells to Th2 cells in patients with hepatocellular carcinoma, potentially promoting antitumor activity. The Th1/Th2 ratio has also been shown to decrease after volatile GA, whereas propofol did not induce any change. These findings show that volatile GA is more likely to suppress anticancer immunity to a greater extent than intravenous GA or RA. ${ }^{1}$

\section{Effects on "Oncogenes," Matrix Metalloproteinase, and Hypoxia inducible factors}

Volatile GA has been shown to modulate gene expression in breast and brain tumor cell cultures, occurring in a unique and timedependent manner. Compared with volatile GA, intravenous GA with RA reduces postoperative IL-1b, suppresses elevation of matrix metalloproteinase (MMP)-3 and MMP-9, and also reduces the increase in hypoxia inducible factor (HIF)-1a and HIF-2a. MMPs are known to be involved in cancer cell invasion, migration, and metastasis. HIF-1a overexpression is associated with poor survival rates and a reduced disease-free rate in colorectal cancer. It has also been shown to reduce response to therapy and shorten disease-free survival in breast cancer patients. Volatile GA has been shown to upregulate HIF1a expression and enhance cancer malignancy. Recent studies have demonstrated that isoflurane upregulates HIF-1a in prostate cancer cells, but propofol downregulates these increases. Both HIF-1a and HIF-2a were upregulated by isoflurane in renal cancer cells as well. Other inhalational anesthetics also upregulate the HIF system, and the possible link to cancer malignancy has been discussed previously. In addition to this, barbiturates have been shown to inhibit HIF-1a activation, whereas local anesthetics used in RA reduced HIF-1a expression. These studies indicate that volatile GA enhances cancer invasion and metastasis to a greater extent than intravenous GA or RA. However, further research, including large clinical trials, are needed prior to concluding that specific anesthetics and techniques influence cancer recurrence postoperatively. ${ }^{1}$

\section{Conclusion}

As of 2013, only one major prospective randomized-control clinical trial has been published on the long-term impact of anesthetic technique and postoperative cancer recurrence. The MASTER trial was a multicenter prospective clinical study which randomized oncologic patients undergoing major abdominal surgery to receive general anesthesia with either epidural or opioid analgesia. No significant difference was detected in cancer free survival. Unfortunately, the amount of volatile anesthetic was not recorded and may represent a 
significant confounding variable. Smaller prospective studies have also been conducted, but like the MASTER trial, they also suffer from the difficulty associated with multimodal anesthesia and the ability of these studies to eliminate confounders and determine the contribution of each individual anesthetic factor to cancer recurrence. Despite the growing body of animal and laboratory evidence, controlled clinical trials are desperately needed to evaluate the impact of each individual anesthetic on cancer recurrence and long-term patient survival. Three multi-center prospective RCTs are on the horizon, however. These trials, looking at tumor recurrence and anesthetic technique for breast, lung, and colon cancers are being conducted by the Cleveland Clinic, Mater University Hospital, and the University of Dusseldorf. Results are expected to be reported in 2018 and 2022. Meanwhile, our patients and anesthesia providers have to wait. Unfortunately, some of our patients do not have the luxury of time. ${ }^{6}$

It is difficult to perform a conclusive comparison between GA and RA due to the huge variety of anesthetic techniques available, as well as the varying anesthetic demands in surgery for a wide range of issues, including malignancy. The current literature looks at specific scenarios and is significantly limited by the lack of applicability to a wider context. There is a strong argument supporting the wider and preferential use of RA and intravenous GA compared to volatile GA in specific circumstances where possible. These might include patients with diagnosed or potentially malignant tumors, or those particularly vulnerable to the surgical stress response. Sufficient evidence to support an immediate alteration of current clinical practice is lacking; however, further research into this area is warranted due to the potential implications elicited by the current research. ${ }^{1}$

\section{Acknowledgments}

None.

\section{Conflicts of interest}

Author declares there are no conflicts of interest.

\section{Funding}

None.

\section{References}

1. Masae I, Matthew E, Atsuhiro S, et al. Anesthesia, surgical stress, and "long-term" outcomes. Acta Anaesthesiol Taiwan. 2015;53(3):99-104.

2. Cruz FF, Rocco PR, Pelosi P. Anti-inflammatory properties of anesthetic agents. Crit Care. 2017;21(1):67.

3. Alan DK, Nayan Patel, Franklin R, et al. Effect of Opiates, Anesthetic Techniques, and Other Perioperative Factors on Surgical Cancer Patients. Ochsner J. 2014;14(2):216-228.

4. Paola S, Mauro B, Leda G, et al. The Effects of Tramadol and Morphine on Immune Responses and Pain After Surgery in Cancer Patients. Anesth Analg. 2000;90(6):1411-1414.

5. Inada $\mathrm{T}$, Yamanouchi $\mathrm{Y}$, Jomura $\mathrm{S}$, et al. Effect of propofol and isoflurane anaesthesia on the immune response to surgery. Anaesthesia. 2004;59(10):954-959.

6. Andres Missair, Ralf EG. Cancer Recurrence and Anesthetic Technique: A "Wake Up Call". Int J Clin Anesthesiol . 2013;1:1005.

7. Colucci DG, Puig NR, Hernandez PR. Influence of anaesthetic drugs on immune response: from inflammation to immunosuppression. OA Anaesthetics. Open Access. 2013;1(3):21.

8. Magdy Ali Omera. Do Volatile Inhalation Anesthetics Modify the Immune Response in Surgical Patients. AJAIC Vol. 2006; (9).

9. Melamed R, Bar Yosef S, Shakhar G, et al. Suppression of natural killer cell activity and promotion of tumor metastasis by ketamine, thiopental, and halothane, but not by propofol: mediating mechanisms and prophylactic measures. Anesth Analg . 2003;97(5):1331-1339.

10. Helmy S, Wahby M, El Nawaway M. The Impact of Anesthesia and Surgery on Plasma Cytokine Production. Anaesthesia. 1999;54(8):733-738.

11. Stollings LM, Jia LJ, Tang P, et al. Immune Modulation by Volatile Anesthetics. Anesthesiology. 2016;125(2):399-411. 